\title{
THE
}

1975

\section{The Interaction of Rare Gas Atoms with Graphite Surfaces. II. Adatom-adatom Potentials}

David L. Freeman

University of Rhode Island, dfreeman@uri.edu

Follow this and additional works at: https://digitalcommons.uri.edu/chm_facpubs

Terms of Use

All rights reserved under copyright.

\section{Citation/Publisher Attribution}

Freeman, D. L. (1975). The Interaction of Rare Gas Atoms with Graphite Surfaces. II. Adatom-Adatom Potentials. J. Chem. Phys., 62(3), 4300-4307. doi: 10.1063/1.430351

Available at: http://dx.doi.org/10.1063/1.430351 


\section{AIP $\begin{gathered}\text { mosoumator } \\ \text { chemical Physics }\end{gathered}$}

The interaction of rare gas atoms with graphite surfaces. II. Adatom-adatom potentials

David L. Freeman

Citation: J. Chem. Phys. 62, 4300 (1975); doi: 10.1063/1.430351

View online: http://dx.doi.org/10.1063/1.430351

View Table of Contents: http://jcp.aip.org/resource/1/JCPSA6/v62/i11

Published by the American Institute of Physics.

Additional information on J. Chem. Phys.

Journal Homepage: http://jcp.aip.org/

Journal Information: http://jcp.aip.org/about/about_the_journal

Top downloads: http://jcp.aip.org/features/most_downloaded

Information for Authors: http://jcp.aip.org/authors

\section{ADVERTISEMENT}

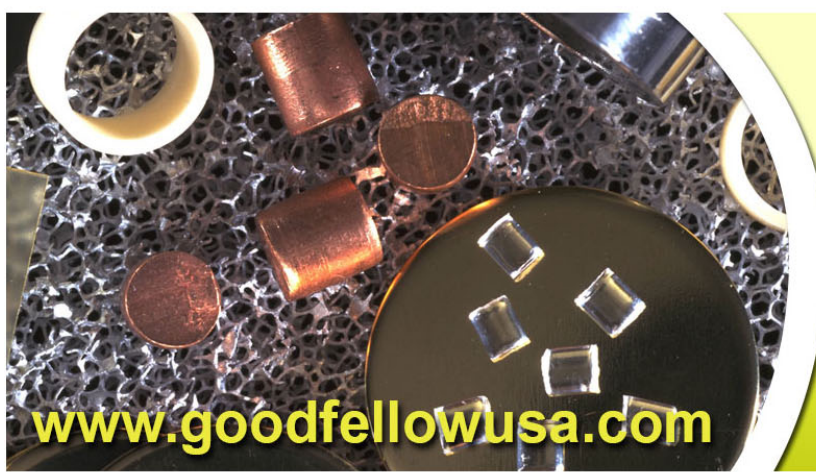




\title{
The interaction of rare gas atoms with graphite surfaces. II Adatom-adatom potentials
}

\author{
David L. Freeman* \\ Battelle Memorial Institute, Columbus, Ohio 43201 \\ (Received 9 December 1974) \\ The Gordon-Kim local density method is applied to the calculation of the interaction energy of pairs of neon, \\ argon, and krypton atoms adsorbed on the basal plane of graphite. At the adatom-surface equilibrium separation, \\ the adatom-adatom interaction potentials are found to be from $12 \%$ to $20 \%$ more repulsive than the gas phase \\ counterparts. At much smaller adatom-surface separations, the adatom-adatom effective potential well depth is \\ only $3 \%$ of the gas phase well depth. It is found that the nonadditive contributions to the interaction energy are \\ more important for the smaller rare gas adatoms than for the larger ones. This trend is observed experimentally. \\ It is concluded that rare gas films on graphite are too mobile to be in registry with the substrate.
}

\section{INTRODUCTION}

A variety of interesting questions concerning the properties of adsorbed gas atoms on the surfaces of solids depends upon the interactions between the adatoms. For example, adsorption isotherms and adsorbate structure are both strongly influenced by adatom-adatom potentials. A study of the general nature of these forces is an important problem.

One of the better studied systems in physisorption is rare gas monolayers on graphite. Rare gas adsorption on graphite has received this attention because the adatom migration barriers are very small, and these highly mobile films are a prototype for studying two-dimensional systems. Some of the more interesting phenomena observed in two dimensional systems are the possible occurrences of phase transitions. Recent experiments of helium monolayers ${ }^{1}$ and xenon monolayers ${ }^{2}$ on graphite at moderate densities and low temperatures have indicated a possible phase transition to an ordered state. There is evidence that the adatoms in this ordered state are in registry with the substrate. An understanding of such an ordered state and its degree of registry requires a knowledge of the interaction potential between the adatoms.

From experimental investigations of the so-called third-order regions of physical adsorption isotherms, it has become clear that adsorbed adatoms interact with each other more repulsively than they do in the gas phase. ${ }^{3-11}$ Recent analysis of the experimental data of rare gas adsorption on graphite surfaces has found that the repulsive interaction due to the surface decreases the adatom-adatom binding energies by $5 \%-10 \%{ }^{8.9}$ To explain this additional repulsion Sinanoğlu and Pitzer calculated the nonadditive contribution to the interaction energy between two nonpolar adatoms and a surface using third-order perturbation theory. ${ }^{12}$ Sinanoğlu and Pitzer predicted that the adatom-adatom well depth would decrease by $20 \%-40 \%$, which is a much larger effect than is actually observed. Additionally, the application of their theory to rare gas adsorption on graphite predicted the adatom-adatom well depth to be lowered more for large atoms than for small ones, and this trend is at variance with experiment. ${ }^{8}$ McLachlan $^{13}$ treated the three-body, two-adatom-surface interaction by applying the method of images. Application of McLachlan's theory to rare gas adsorption on graphite predicted well depth lowerings of the right order of magnitude, but again predicted the wrong trend with respect to adatom size. $^{8}$ As MacRury and Linder ${ }^{14}$ have pointed out, one of the basic shortcomings of both the theories of Sinanoglu and Pitzer and of McLachlan is that they treated the surface as a continuous, structureless medium, and thus did not incorporate any local effects. To analyze the importance of the local properties of the substrate, MacRury and Linder computed the nonadditive contribubutions to the dispersion energy for rare gas adsorption on xenon crystals. By comparing their results with the continuum model of McLachlan, they found the continuum model to predict a somewhat smaller nonadditive contribution. 14

All three of these theoretical treatments are valid only when there is no significant overlap between the electronic charge distributions of the adatoms, and between the electronic charge distributions of the adatoms and the surface. Since there is large overlap for equilibrium geometries in physisorption, the nonoverlap approximation may be expected to introduce serious errors.

In a recent paper ${ }^{15}$ (hereafter referred to as I), we applied the Gordon-Kim (GK) local density method ${ }^{16}$ to the calculation of the interaction energy of single rare-gas atoms and a graphite surface. The GK method has the advantage of incorporating the effect of the local environment of the surface, and it is valid when there is significant overlap between the electronic charge distributions. In what follows we apply the GK method to calculate the nonadditive contribution to the adatom-adatom interactions for rare gas adsorption on graphite. As we pointed out in $I$, the GK method does not include long-range dispersion effects correctly. Recently, Kim calculated the three-body contribution to the interaction energy for $\mathrm{He}_{3}, \mathrm{Ne}_{3}, \mathrm{Ar}_{3}$, and $\mathrm{Kr}_{3}$ both with and without dispersion effects. ${ }^{17} \mathrm{He}$ found that from the repulsive region to the equilibrium configuration, the dispersion contribution amounted to less than $15 \%$ of the total three-body energy. Since we deal with an infinite system in the calculations which follow, the dispersion error will be worse for our case than for the three atom case studied by Kim. However there will also be a dispersion error in the total energy, and we expect the 
relative importance of the nonadditive energy to be well described.

In Sec. II we develop the necessary extensions of the GK method to compute the nonadditive contributions to rare gas adsorption on graphite. In Sec. III we present our results, and our conclusions are given in Sec. IV.

\section{APPLICATION OF THE GORDON-KIM METHOD TO ADATOM-ADATOM INTERACTIONS}

The details of the GK method and its application to the physisorption of rare gas atoms on graphite are given in I. The notation and the electron density contributions for the adatoms and for the graphite surface used in this work are the same as in I. As Bennett ${ }^{18}$ and $\mathrm{Kim}^{17}$ have pointed out, the Coulombic contribution to the interaction energy is two-body additive, i. e., we can write

$$
V_{c}\left(\mathbf{R}_{a}, \mathbf{R}_{b}\right)=V_{c}\left(\mathbf{R}_{a}\right)+V_{c}\left(\mathbf{R}_{b}\right)+V_{c}\left(\mathbf{R}_{a b}\right),
$$

where $V_{c}\left(\mathbf{R}_{a}, \mathbf{R}_{b}\right)$ is the Coulombic contribution to the interaction energy of the two-atom-surface system, $\mathbf{R}_{\mathbf{a}}$ and $\mathbf{R}_{b}$ are, respectively, the coordinates of adatoms $a$ and $b$ relative to the graphite surface, $\mathbf{R}_{a b}$ is the adatom-adatom internuclear separation, $V_{c}\left(\mathbf{R}_{a}\right)$ is given by Eq. (7) of I, and

$$
V_{c}\left(\mathbf{R}_{a b}\right)=\int \frac{\rho_{a}(\mathbf{r}) \rho_{b}\left(\mathbf{r}^{\prime}\right)}{\left|\mathbf{r}-\mathbf{r}^{\prime}\right|} d^{3} r d^{3} r^{\prime} .
$$

In Eq. (2), $\rho_{a}(\mathbf{r})$ and $\rho_{b}(\mathbf{r})$ are, respectively, the charge distributions (including the nuclei) of adatoms $a$ and $b$. We write the total interaction energy $E\left(\mathbf{R}_{a}, \mathbf{R}_{b}\right)$ for the two-adatom-surface system as

$$
E\left(\mathbf{R}_{a}, \mathbf{R}_{b}\right)=E\left(\mathbf{R}_{a}\right)+E\left(\mathbf{R}_{b}\right)+E\left(\mathbf{R}_{a b}\right)+\Delta E\left(\mathbf{R}_{a}, \mathbf{R}_{b}\right)
$$

where $E\left(\mathbf{R}_{a}\right)$ is given by Eq. (17) of $\mathrm{I}$, and $E\left(\mathbf{R}_{a b}\right)$ is the GK gas phase atom-atom interaction energy. Using Eq. (1) the nonadditive contribution $\Delta E\left(\mathbf{R}_{a}, \mathbf{R}_{b}\right)$ is given by

$$
\Delta E\left(\mathbf{R}_{a}, \mathbf{R}_{b}\right)=E_{g}\left(\mathbf{R}_{a}, \mathbf{R}_{b}\right)-E_{g}\left(\mathbf{R}_{a}\right)-E_{g}\left(\mathbf{R}_{b}\right)-E_{g}\left(\mathbf{R}_{a b}\right) .
$$

In Eq. (4), $E_{g}(\mathbf{R})$ is defined by Eq. (13) of I and

$$
E_{g}\left(\mathbf{R}_{a}, \mathbf{R}_{b}\right)=\int d^{3} r \bar{\rho}_{T}(\mathbf{r}) E_{G}\left(\bar{\rho}_{T}(\mathbf{r})\right),
$$

where

$$
\bar{\rho}_{T}(\mathbf{r})=\bar{\rho}_{a}(\mathbf{r})+\bar{\rho}_{b}(\mathbf{r})+\bar{\rho}_{s}(\mathbf{r})
$$

In Eq. (15), $E_{G}(\rho)$ is defined by Eq. (14) of I. As in I, the bar over the $\rho$ denotes the electronic contribution to the charge density. If we evaluate all terms of Eq. (4) in the same integral, we have

$$
\begin{aligned}
\Delta E\left(\mathbf{R}_{a}, \mathbf{R}_{b}\right)= & \int d^{3} r\left\{\bar{\rho}_{T}(\mathbf{r}) E_{G}\left[\bar{\rho}_{T}(\mathbf{r})\right]-\left[\bar{\rho}_{a}(\mathbf{r})+\bar{\rho}_{b}(\mathbf{r})\right] E_{G}\left[\bar{\rho}_{a}(\mathbf{r})+\bar{\rho}_{b}(\mathbf{r})\right]-\left[\bar{\rho}_{a}(\mathbf{r})+\bar{\rho}_{s}(\mathbf{r})\right] E_{G}\left[\bar{\rho}_{a}(\mathbf{r})+\bar{\rho}_{s}(\mathbf{r})\right]\right. \\
& \left.-\left[\bar{\rho}_{b}(\mathbf{r})+\bar{\rho}_{s}(\mathbf{r})\right] E_{G}\left[\bar{\rho}_{b}(\mathbf{r})+\bar{\rho}_{s}(\mathbf{r})\right]+\bar{\rho}_{a}(\mathbf{r}) E_{G}\left[\bar{\rho}_{a}(\mathbf{r})\right]+\bar{\rho}_{b}(\mathbf{r}) E_{G}\left[\bar{\rho}_{b}(\mathbf{r})\right]+\bar{\rho}_{s}(\mathbf{r}) E_{G}\left[\bar{\rho}_{s}(\mathbf{r})\right]\right\}
\end{aligned}
$$

Using the fact that $\bar{\rho}_{s}(\mathbf{r})$ is periodic over the two-dimensional graphite lattice, we have

$$
\Delta E\left(\mathbf{R}_{a}, \mathbf{R}_{b}\right)=\sum_{\mu} K_{\mu}\left(\mathbf{R}_{a}, \mathbf{R}_{b}\right),
$$

where

$$
\begin{aligned}
K_{\mu}\left(\mathbf{R}_{a}, \mathbf{R}_{b}\right)= & \int_{c} d^{3} r\left\{\bar{\rho}_{T}\left(\mathbf{r}+\mathbf{R}_{\mu}\right) E_{G}\left[\bar{\rho}_{T}\left(\mathbf{r}+\mathbf{R}_{\mu}\right)\right]-\left[\bar{\rho}_{a}\left(\mathbf{r}+\mathbf{R}_{\mu}\right)+\bar{\rho}_{b}\left(\mathbf{r}+\mathbf{R}_{\mu}\right)\right] E_{G}\left[\bar{\rho}_{a}\left(\mathbf{r}+\mathbf{R}_{\mu}\right)+\bar{\rho}_{b}\left(\mathbf{r}+\mathbf{R}_{\mu}\right)\right]\right. \\
& -\left[\bar{\rho}_{a}\left(\mathbf{r}+\mathbf{R}_{\mu}\right)+\bar{\rho}_{s}(\mathbf{r})\right] E_{G}\left[\bar{\rho}_{a}\left(\mathbf{r}+\mathbf{R}_{\mu}\right)+\bar{\rho}_{s}(\mathbf{r})\right]-\left[\bar{\rho}_{b}\left(\mathbf{r}+\mathbf{R}_{\mu}\right)+\bar{\rho}_{s}(\mathbf{r})\right] E_{G}\left[\bar{\rho}_{b}\left(\mathbf{r}+\mathbf{R}_{\mu}\right)+\bar{\rho}_{s}(\mathbf{r})\right] \\
& \left.+\bar{\rho}_{a}\left(\mathbf{r}+\mathbf{R}_{\mu}\right) E_{G}\left[\bar{\rho}_{a}\left(\mathbf{r}+\mathbf{R}_{\mu}\right)\right]+\bar{\rho}_{b}\left(\mathbf{r}+\mathbf{R}_{\mu}\right) E_{G}\left[\bar{\rho}_{b}\left(\mathbf{r}+\mathbf{R}_{u}\right)\right]+\bar{\rho}_{s}(\mathbf{r}) E_{G}\left[\bar{\rho}_{s}(\mathbf{r})\right]\right\} .
\end{aligned}
$$

The $c$ of Eq. (9) implies integration over one unit cell, and $R_{\mu}$ is a lattice vector. As in the case of single adatom energies, each $K_{\mu}$ contribution was evaluated in Cartesian coordinates using the same quadrature schemes that were used in I. The terms of Eq. (8) fall off exponentially, and its convergence is rapid. We found that by summing over all unit cells in Eq. (8) within 10 a. $u$. of the projected position of the center of $\mathbf{R}_{a b}$ onto the plane of the graphite surface, we attained convergence to three significant figures.

The nonadditive contribution to the interaction energy given in Eq. (8) is a property of the full two-atom-surface system, and it is not possible to uniquely define nonadditive contributions to the adatom-adatom interaction potentials. However, it is usually assumed that the adatom-surface interaction is unperturbed by the presence of the second adatom, and the nonadditive contribution is incorporated into an effective adatom-ad-

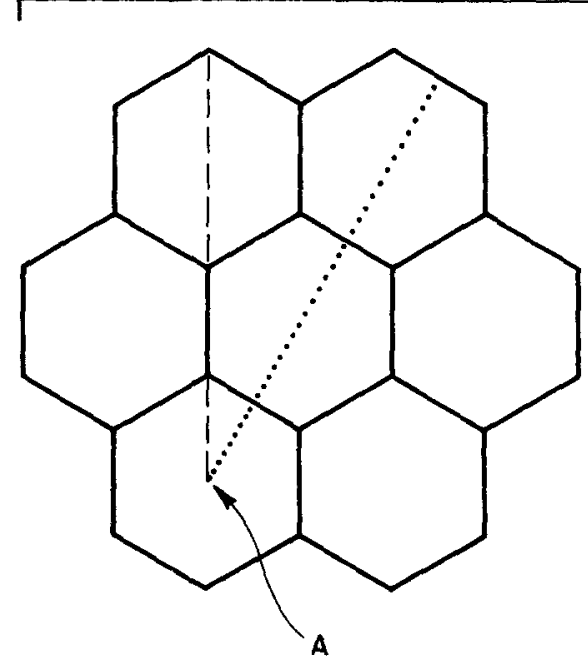

FIG. 1. A section of the graphite lattice is shown along with the two paths used in this calculation. 


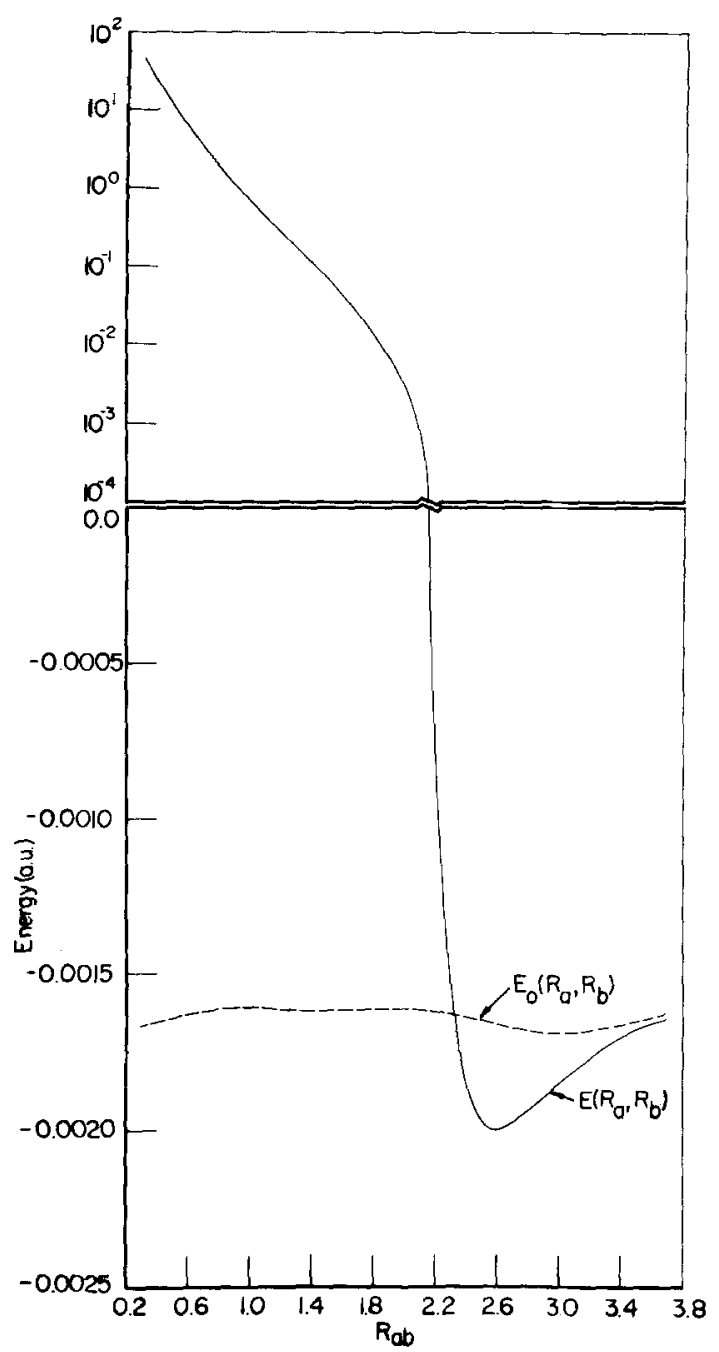

FIG. 2. The total energy as a function of adatom-adatom separation for two argon atoms at 6.6 a. u. above the graphite surface oriented along the dashed line of Fig. 1 is shown in the solid line. The dashed line is the total energy in the limit of no argon-argon interaction. Distances are measured in units of a graphite lattice carbon-carbon bond length.

atom interaction. We define this effective interaction $V_{\text {eqf }}\left(\mathbf{R}_{a}, \mathbf{R}_{b}\right)$ by

$$
V_{\text {eIf }}\left(\mathbf{R}_{a}, \mathbf{R}_{b}\right)=E\left(\mathbf{R}_{a b}\right)+\Delta E\left(\mathbf{R}_{a}, \mathbf{R}_{b}\right) .
$$

In the calculations which follow we compare $V_{\text {eft }}$ with the gas phase atom-atom potentials to examine the changes induced by the graphite surface.

\section{RESULTS}

We expect physisorbed rare gas atoms on graphite to be found at their single adatom potential energy minima with respect to the adatom-surface vertical distance. In I we found very small migration barriers with respect to motion parallel to the plane of the graphite surface, and any relative orientation of the two adatoms is likely. Using Eqs. (3), (8), and (10), we computed the interaction energy of two argon atoms both at $6.6 \mathrm{a} . \mathrm{u}$. above the graphite surface. One argon atom was kept fixed at position A of Fig. 1, and the energy was computed as a function of the position of the other adatom as it moved
TABLE I. The interaction between two argon atoms 6.6 a. u. above the graphite surface oriented along the dashed line of Fig. 1."

\begin{tabular}{llllll}
\hline$R_{a b}$ & $E\left(\mathrm{R}_{a b}\right)$ & $\Delta E\left(\mathrm{R}_{a}, \mathrm{R}_{b}\right)$ & $V_{\text {eft }}\left(\mathrm{R}_{a}, \mathrm{R}_{b}\right)$ & $V_{2 B}\left(\mathrm{R}_{a}, \mathrm{R}_{b}\right)$ & $E\left(\mathrm{R}_{a}, \mathrm{R}_{b}\right)$ \\
\hline 0.3 & 45.6 & 0.000831 & 45.6 & 45.6 & 45.6 \\
0.6 & 5.55 & 0.000728 & 5.55 & 5.55 & 5.55 \\
0.9 & 1.03 & 0.000596 & 1.03 & 1.03 & 1.03 \\
1.0 & 0.654 & 0.000550 & 0.655 & 0.652 & 0.653 \\
1.2 & 0.278 & 0.000459 & 0.278 & 0.276 & 0.277 \\
1.5 & 0.0724 & 0.000331 & 0.0727 & 0.0708 & 0.0711 \\
1.8 & 0.0153 & 0.000222 & 0.0155 & 0.0137 & 0.0139 \\
2.0 & 0.00435 & 0.000163 & 0.00451 & 0.00274 & 0.00290 \\
2.1 & 0.00200 & 0.000138 & 0.00214 & 0.000389 & 0.000527 \\
2.2 & 0.000704 & 0.000116 & 0.000820 & -0.000912 & -0.000796 \\
2.3 & 0.0000343 & 0.0000967 & 0.000131 & -0.00159 & -0.00149 \\
2.35 & -0.000152 & 0.0000880 & -0.0000640 & -0.00178 & -0.00169 \\
2.4 & -0.000273 & 0.0000799 & -0.000193 & -0.00191 & -0.00183 \\
2.45 & -0.000347 & 0.0000725 & -0.000275 & -0.00199 & -0.00191 \\
2.5 & -0.000385 & 0.0000656 & -0.000319 & -0.00203 & -0.00196 \\
2.6 & -0.000394 & 0.0000534 & -0.000341 & -0.00205 & -0.002610 \\
2.65 & -0.000378 & 0.0000481 & -0.000330 & -0.00204 & -0.00199 \\
2.7 & -0.000354 & 0.0000432 & -0.000311 & -0.002012 & -0.00198 \\
2.8 & -0.000298 & 0.0000348 & -0.000263 & -0.00198 & -0.00194 \\
2.9 & -0.000239 & 0.0000278 & -0.000211 & -0.00193 & -0.00190 \\
3.0 & -0.000186 & 0.0000221 & -0.000164 & -0.00188 & -0.00185 \\
3.1 & -0.000141 & 0.0000176 & -0.000123 & -0.00183 & -0.00181 \\
3.4 & -0.0000556 & 0.00000868 & -0.0000469 & -0.00171 & -0.00170 \\
3.7 & -0.0000204 & 0.00000413 & -0.0000163 & -0.00164 & -0.00164 \\
\hline \hline & - & & & &
\end{tabular}

All numbers expressed in $\mathrm{a}_{0} \mathrm{u}$. except $R_{a b}$ which is given in units of a two-dimensional graphite carbon-carbon bond length.

along the direction of the dashed line in Fig. 1. The results are given in Table 1. The total energy is plotted in Fig. 2, and the effective adatom-adatom interaction as well as the gas phase argon-argon GK potential are plotted in Fig. 3. In Table I and in Figs. 1 and 2 the adatom-adatom distance is measured in units of the carbon-carbon bond length of the two-dimensional graphite lattice (1.42 $\AA$ ), and the energies are given in atomic units. In Table I we have also given the two-body addi-

TABLE II. The interaction between two argon atoms 6.6 a.u. above the graphite surface oriented along the dotted line of Fig.

\begin{tabular}{|c|c|c|c|c|c|}
\hline$R_{a b}$ & $E\left(\mathbf{R}_{a b}\right)$ & $\Delta E\left(\mathbf{R}_{a}, \mathbf{R}_{b}\right)$ & $V_{\text {eft }}\left(\mathbf{R}_{a}, R_{b}\right)$ & $V_{2 B}\left(\mathbf{R}_{\sigma}, \mathbf{R}_{\partial}\right)$ & $E\left(\mathrm{R}_{a}, \mathrm{R}_{b}\right)$ \\
\hline 0.866 & 1.21 & 0.000545 & 1.21 & 1.21 & 1.21 \\
\hline 1.04 & 0.551 & 0.000532 & 0.552 & 0.549 & 0.550 \\
\hline 1.20 & 0.278 & 0.000459 & 0.278 & 0.276 & 0.277 \\
\hline 1.21 & 0.264 & 0.000454 & 0.264 & 0.262 & 0.262 \\
\hline 1.39 & 0.123 & 0.000378 & 0.123 & 0.121 & 0.121 \\
\hline 1.56 & 0.0544 & 0.000309 & 0.0547 & 0.0527 & 0.0530 \\
\hline 1.65 & 0.0352 & 0.000276 & 0.0355 & 0.0335 & 0.0338 \\
\hline 1.73 & 0.0223 & 0.000246 & 0.0225 & 0.0206 & 0.0208 \\
\hline 1.82 & 0.0137 & 0.000217 & 0,0139 & 0.0120 & 0.0122 \\
\hline 1.91 & 0.00815 & 0.000191 & 0,00834 & 0.00647 & 0.00666 \\
\hline 2.08 & 0.00240 & 0.000144 & 0.00254 & 0.000730 & 0.000874 \\
\hline 2.17 & 0.00107 & 0.000124 & 0.00119 & -0.000584 & -0.000460 \\
\hline 2.25 & 0.000301 & 0.000106 & 0,000407 & -0.00134 & -0.00123 \\
\hline 2.28 & 0.000130 & 0.000101 & 0.000231 & -0.00151 & -0.00141 \\
\hline 2.30 & 0.0000343 & 0.0000974 & 0.000132 & -0.00160 & -0.00151 \\
\hline 2.31 & $-0,00000677$ & 0.0000957 & 0.0000889 & -0.00164 & -0.00155 \\
\hline 2.34 & -0.000115 & 0.0000906 & -0.0000244 & -0.00175 & -0.00166 \\
\hline 2.40 & -0.000266 & 0.0000810 & -0.000185 & -0.00189 & -0.00181 \\
\hline 2.42 & -0.000315 & 0.0000766 & -0.000238 & -0.00195 & -0.00187 \\
\hline 2.51 & $-0,000390$ & 0.0000643 & -0.000326 & -0.00201 & $-0,00195$ \\
\hline 2,54 & -0.000398 & 0.0000606 & -0.000337 & -0.00202 & -0.00196 \\
\hline 2.59 & -0.000395 & 0.0000537 & -0.000341 & -0.00202 & -0.00196 \\
\hline 2.60 & -0.000394 & 0.0000535 & -0.000341 & -0.00201 & -0.00196 \\
\hline 2.66 & -0.000376 & 0.0000474 & -0.000329 & & \\
\hline 2.77 & -0.000314 & 0.0000368 & -0.000277 & -0.00194 & -0.00191 \\
\hline 2.94 & -0.000214 & 0.0000247 & -0.000189 & -0.00185 & $-0,00183$ \\
\hline 3.00 & -0.000186 & 0.0000217 & -0.000164 & -0.00183 & -0.00181 \\
\hline 3.12 & -0.000134 & 0.0000165 & -0.000118 & -0.00180 & -0.00179 \\
\hline 3.29 & -0.0000791 & 0.0000110 & $-0,0000681$ & -0.00176 & -0.00175 \\
\hline 3.38 & -0.0000598 & 0.00000896 & -0.0000508 & -0.00175 & -0.00174 \\
\hline 3.46 & -0.0000449 & 0.00000728 & -0.0000376 & -0.00173 & -0.00172 \\
\hline
\end{tabular}

${ }^{2}$ All numbers expressed in a. u. except $R_{a b}$ which is given in units of a two-dimensional graphite carbon-carbon bond length. 


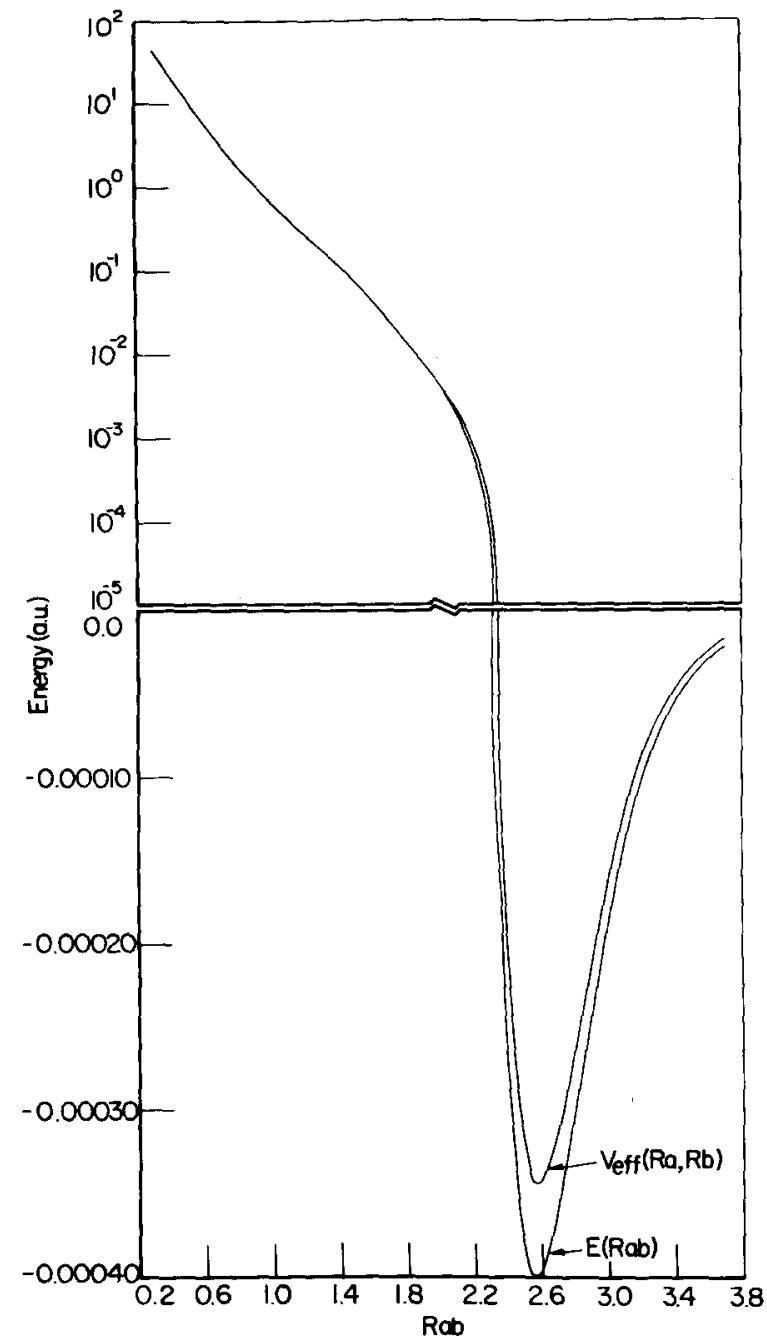

FIG. 3. The gas phase and effective argon-argon potentials are shown. The argon atoms are at 6.6 a.u. above the graphite surface oriented along the dashed line of Fig. 1. The distances are given in units of a graphite lattice carbon-carbon bond length.

tive energy $V_{2 B}\left(\mathbf{R}_{a}, \mathbf{R}_{b}\right)$ defined by

$$
V_{2 B}\left(\mathbf{R}_{a}, \mathbf{R}_{b}\right)=E\left(\mathbf{R}_{a}\right)+E\left(\mathbf{R}_{b}\right)+E\left(\mathbf{R}_{a b}\right) .
$$

From Figs. 1 and 2 we see that the argon-argon distance at equilibrium is the same as in the gas phase. This is a consequence of the single adatom migration barrier being much smaller than the $\mathrm{Ar}_{2}$ effective potential binding energy. In Fig. 2 the dashed line is the sum of the single adatom energies, $E_{0}$, defined by

$$
E_{0}\left(\mathbf{R}_{a}, \mathbf{R}_{b}\right)=E\left(\mathbf{R}_{a}\right)+E\left(\mathbf{R}_{b}\right) .
$$

It is clear that $E_{0}$ is the energy in the limit of no $\mathrm{Ar}_{2}$ interaction, and the oscillations in $E_{0}$ seen in Fig. 2 reflect the single adatom migration barriers. From Fig. 3 we see that the equilibrium configuration predicted by $E\left(\mathbf{R}_{a b}\right)$ and $V_{\text {ofs }}\left(\mathbf{R}_{a}, \mathbf{R}_{\partial}\right)$ are the same to within the accuracy of the calculation. We find that the well depth is decreased by $13 \%$ owing to the nonadditive effects. This compares well with the decrease of $10.2 \%$ reported by Everett. $^{8}$
As we have indicated, the smoothness of a graphite surface makes it necessary to consider other relative orientations of the two adatoms. In Table II we give the energy as a function of the argon-argon distance for two argon atoms at 6.6 a. u. above the surface, where one argon atom is kept at position $\mathrm{A}$ of $\mathrm{Fig} .1$ and the other is moved along the dotted line of Fig. 1. The values computed at argon-argon separations of 1.2, 2.3, 2.6, and 3.0 units of a graphite carbon-carbon bond length were exactly the same separations as used in Table I. By comparing the nonadditive contributions given in Tables I and II at these four points we see that they are essentially the same, and it is clear that the effective potential is nearly orientation independent at the equilibrium adatom-surface distance of 6.6 a. u. However, the differences in the total energies at these four points are significant, and these differences reflect the changes owing to the argon-argon orientation with respect to the graphite surface. Figure 4 is a plot of the total energy as a function of the argon-argon distance

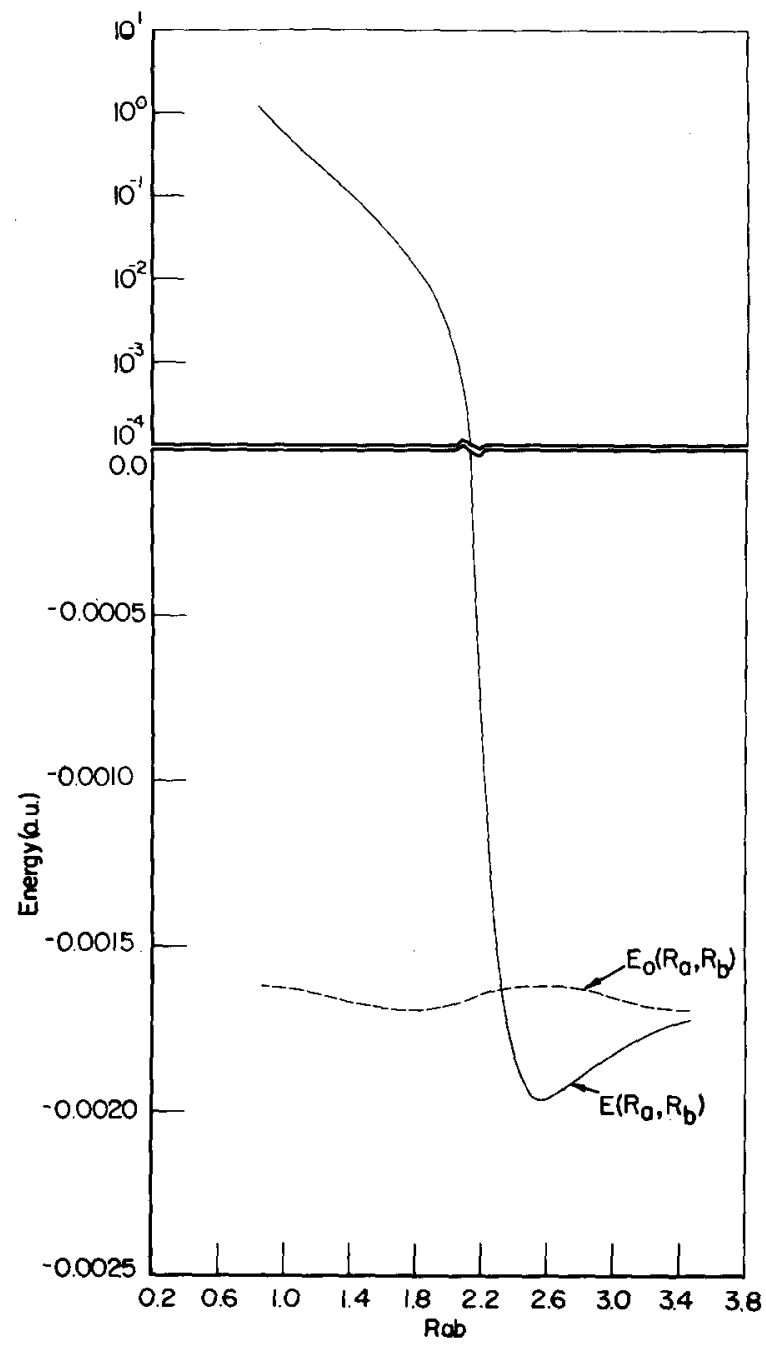

FIG. 4. The total energy as a function of adatom-adatom separation for two argon atoms at 6.6 a. u. above the graphite surface oriented along the dotted line of Fig. 1 is shown in the solid line. The dashed line is the total energy in the limit of no argon-argon interaction. Distances are measured in units of a graphite lattice carbon-carbon bond length. 


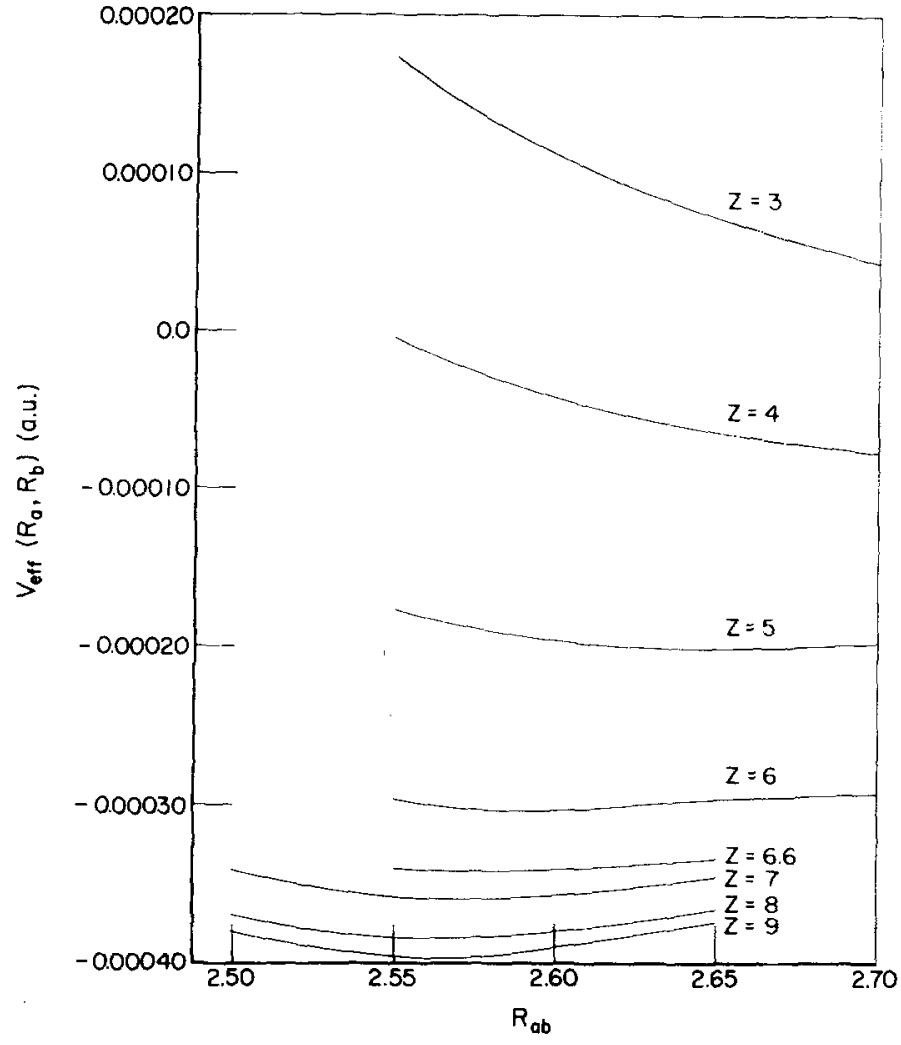

FIG. 5. The effective potential for $\mathrm{Ar}_{2}$ oriented along the dashed line of Fig. 1 as a function of adatom-adatom separation $R$, and adatom-surface distance $Z$ near the gas phase argon-argon equilibrium separation. The adatom-adatom separation is measured in units of a graphite lattice carboncarbon bond length and the adatom-surface distance is measured in a.u.

taken from Table II. As with Fig. 2, the potential minimum occurs at the same point as in the gas phase, but the minimum energy is about $25 \mathrm{cal} / \mathrm{mole}$ higher for the dotted path of Fig. 1 than it is for the dashed path. This very small difference is another measure of the smoothness of the surface.

It is also of interest to examine the nonadditive contributions to adatom-adatom interactions as a function of the adatom-surface distance. In Fig. 5 we plot $V_{e f f}\left(R_{a}, \mathbf{R}_{b}\right)$ for four points near the gas phase argon-argon separation as a function of the distance between the adatoms and the surface. The orientation of the two argon atoms was taken to be along the dashed line of Fig. 1. We note that there is little change of the equilibrium geometry except in the repulsive region very near to the graphite surface. To examine the behavior of two adatoms interacting very close to the surface in more detail, we computed a potential curve for $\mathrm{Ar}_{2}$ at 3. 0 a. u. above the graphite surface. Again the adatoms were oriented along the dashed line of Fig. 1. The results are given in Table 11 . In the repulsive $\mathrm{Ar}_{2}$ region the nonadditive contribution is negative and it becomes positive as the two argon atoms are separated from each other. Kim found the same behavior for clusters of three rare gas atoms in the strongly repulsive regions. ${ }^{17}$ In Fig. 6 we plot the total interaction energy and the
TABLE III. The interaction between two argon atoms 3 a.u. above the graphite surface oriented along the dashed line of Fig. 1. ${ }^{\mathrm{a}}$

\begin{tabular}{|c|c|c|c|c|c|}
\hline$R_{a b}$ & $E\left(\mathrm{R}_{a b}\right)$ & $\Delta E\left(\mathbf{R}_{a}, \mathrm{R}_{b}\right)$ & $V_{\text {etf }}\left(\mathrm{R}_{a}, \mathrm{R}_{b}\right)$ & $V_{2 b^{\prime}}\left(\mathrm{R}_{a}, \mathrm{R}_{b}\right)$ & $E\left(\mathbf{R}_{a}, \mathrm{R}_{8}\right)$ \\
\hline 1.0 & 0.654 & -0.0121 & 0.642 & 1.159 & 1.147 \\
\hline 1.1 & 0.425 & -0.00854 & 0.416 & 0.928 & 0.919 \\
\hline 1.2 & 0.278 & -0.00559 & 0.272 & 11.777 & 11.771 \\
\hline 1.5 & 0.0724 & -0.000128 & $0.072: 3$ & $0,5,59$ & 11.559 \\
\hline 1.6 & 0.0444 & 0.010719 & 0.0451 & 0.5332 & 0.0 .333 \\
\hline 1.8 & 0.0153 & 0.00145 & (1. 0168 & 11.514 & $11.511 ;$ \\
\hline 1.9 & 0.00842 & 0.00150 & 0.00992 & 0.511 & 0.311 \\
\hline 2.0 & 0.00435 & $0.01014: 3$ & 0.00578 & 0.309 & $11 . ; 11$ \\
\hline 2.1 & 0.00200 & 0.00130 & 0.00330 & 0.500 & $11.504 t$ \\
\hline 2.2 & 0.000704 & $\{10(111:\}$ & 0.60183 & 0.498 & (1. 499 \\
\hline 2.3 & 0.0000343 & 0.000953 & 0.000987 & $13.4 \times 7$ & 1). $1.4 \mathrm{M}$ \\
\hline 2.4 & -0.000273 & 0.000786 & 0.000513 & 0.47 .1 & $0 .+7.4$ \\
\hline 2.5 & -0.000385 & 0.0006365 & 0.000251 & $11 .+61$ & 11.161 \\
\hline 2.55 & -0.000399 & 0.000569 & 0.000170 & $11,45,3$ & 0.453 \\
\hline 2.6 & -0.000394 & 0.000506 & 0.000112 & 0,147 & 0.446 \\
\hline 2.65 & -0.000378 & 0.000449 & 0.0000710 & 11.440 & 10.7 .40 \\
\hline 2.7 & -0.000354 & 0.000396 & 0.0000420 & 0.434 & 0.434 \\
\hline 2.75 & -0.000327 & 0.000349 & 0.0000220 & & \\
\hline 2.8 & -0.000298 & 0.0003065 & 0.0000080 & 10.424 & 1). 424 \\
\hline 2.85 & -0.000268 & 0.000268 & 0.000000 & 0.421 & 11.421 \\
\hline 2.9 & -0.000239 & 0.000234 & -0.000005 & 0.418 & $0.41 x$ \\
\hline 2.95 & -0.000211 & 0.000203 & -0.000008 & 0.417 & (1. 417 \\
\hline 3.0 & -0.000186 & 0.000176 & -0.0000100 & 0,411 & $0.4 i t i$ \\
\hline 3.1 & -0.000141 & 0.000131 & -0.0000100 & 11.419 & 0.418 \\
\hline 3.2 & -0.000105 & 0.0000966 & -0.0000084 & & \\
\hline 3.3 & -0.0000769 & 0.0000704 & -0.0000065 & $0.4: 3$ & $0,+11 ;$ \\
\hline 3.4 & $-0,0000556$ & 0.0000510 & -0.0000046 & 0.445 & $(1.415)$ \\
\hline
\end{tabular}

${ }^{2}$ All numbers expressed in a.u. except $R_{a b}$ which is given in units of a two-dimensional graphite carbon-carbon bond length.

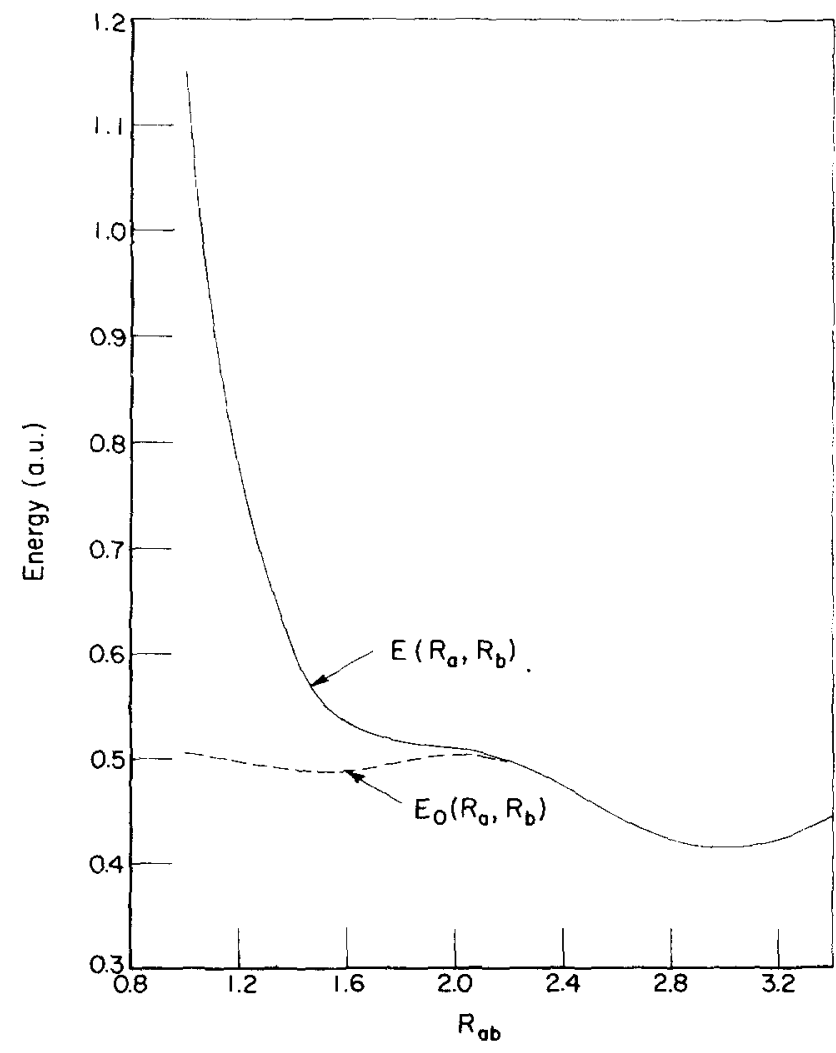

FIG. 6. The total energy as a function of adatom-adatom separation for two argon atoms at 3.0 a. u. above the graphite surface oriented along the dashed line of Fig. 1 is shown in the solid line. The dashed line is the total energy in the limit of no argon-argon interaction. Distances are measured in units of a graphite lattice carbon-carbon bond length. 




FIG. 7. The gas phase and effective argon-argon potentials are shown. The argon atoms are at 3.0 a.u. above the graphite surface oriented along the dashed line of Fig. 1. The distances are given in units of a carbon-carbon bond length.

sum of the single atom energies. The migration barriers are larger than the $\mathrm{Ar}_{2}$ binding energy for this case, and the minimum energy occurs when both adatoms are above the center of a hexagon of the graphite lattice. Of course, the total energy is still net repulsive. In Fig. 7 we plot the effective argon-argon potential for this case along with the gas phase $\mathrm{Ar}_{2}$ GK potential. The minimum of the effective potential occurs at 8.05 a. u. , which is the same point as the minimum of the total energy. This minimum is clearly larger than the gas phase minimum. The well depth of the effective potential is only $3 \%$ of the gas phase well depth, indicating the importance of nonadditive interactions to the effective potential. However, the nonadditive energy is only a small fraction of the total energy near equilibrium.

We also examined neon-neon interactions over graphite. The neon atoms were oriented along the dashed line of Fig. 1 and kept at the equilibrium atom-surface distance of 6.0 a. u. The total energy for the neon-neon interaction is plotted in Fig. 8, and the effective potential along with the gas phase GK potential is plotted in Fig. 9. As was the case with argon on graphite, the equilibrium geometry gives the same neon-neon separation as found in the gas phase. The $\mathrm{Ne}_{2}$ interaction energy becomes small more quickly than the $\mathrm{Ar}_{2}$ case, and the tail of the total energy potential curve shows oscil-

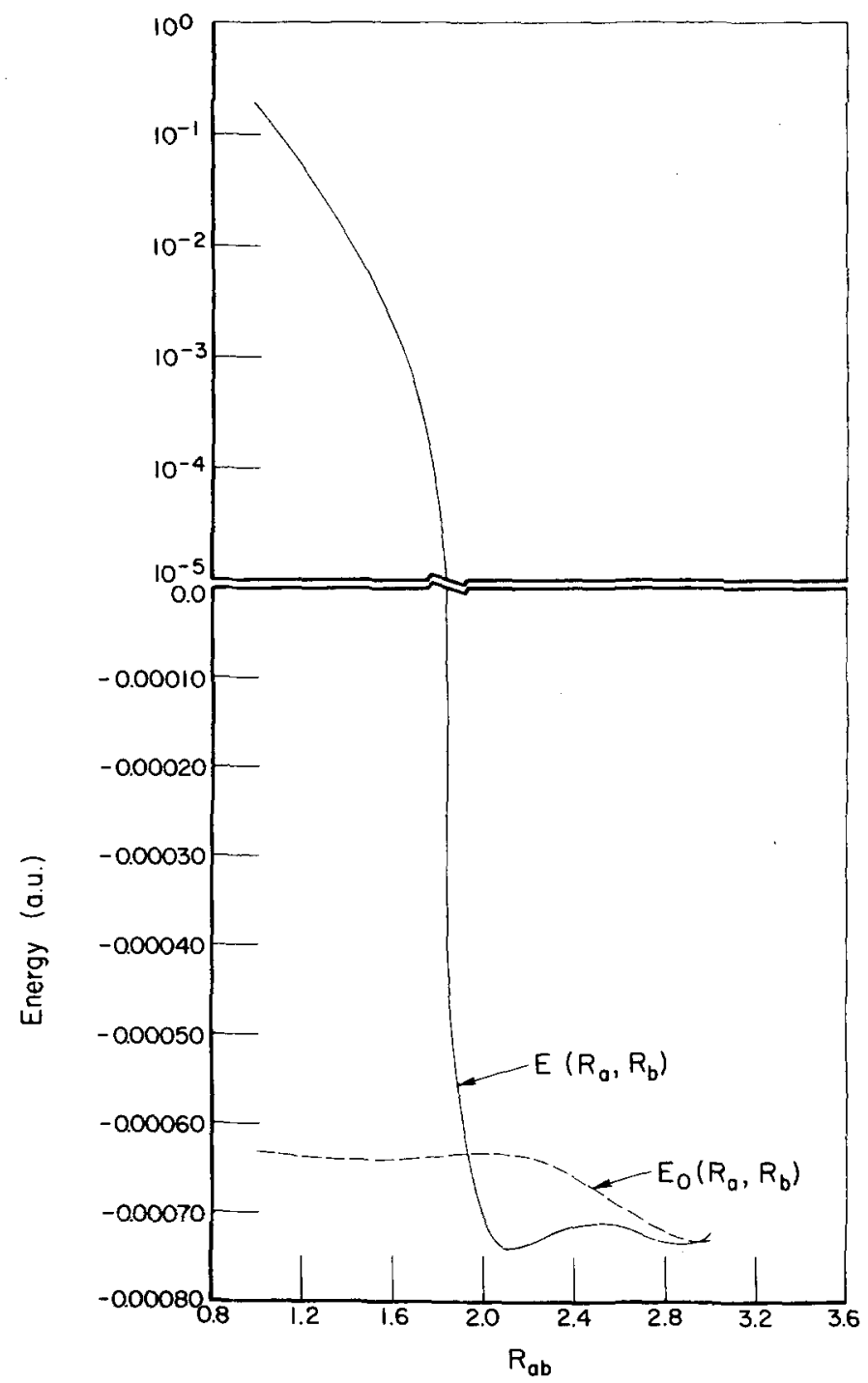

FIG. 8. The total energy as a function of adatom-adatom separation for two neon atoms $6.0 \mathrm{a} . \mathrm{u}$. above the graphite surface oriented along the dashed line of Fig. 1 is shown in the solid line. The dashed line is the total energy in the limit of no neon-neon interaction. Distances are measured in units of a carbon-carbon bond length. 
lations owing to the migration barrier on the graphite lattice. For neon on graphite the well depth of the effective potential is $21 \%$ lower than the gas phase potential. This is the correct trend with respect to adatom size. $^{8}$

To examine the trends more completely, we also performed calculations for krypton on graphite. The effective potential and gas phase GK potential are plotted in Fig. 10. Again the minima of both the gas phase potential and the effective potential occur at the same krypton-krypton separation. The effective potential minimum is $12 \%$ lower than the gas phase minimum.

Following Everett ${ }^{8}$ we can define the ratio of the decrease in the well depth of the effective potential to the gas phase potential by

$$
\Delta=\left[E\left(\mathbf{R}_{a b_{0}}\right)-V_{a t t}\left(\mathbf{R}_{a_{0}}, \mathbf{R}_{b_{0}}\right)\right] /\left[E\left(\mathbf{R}_{a b_{0}}\right)\right],
$$

where $\mathbf{R}_{a b_{0}}$ is the gas phase minimum energy atom-atom

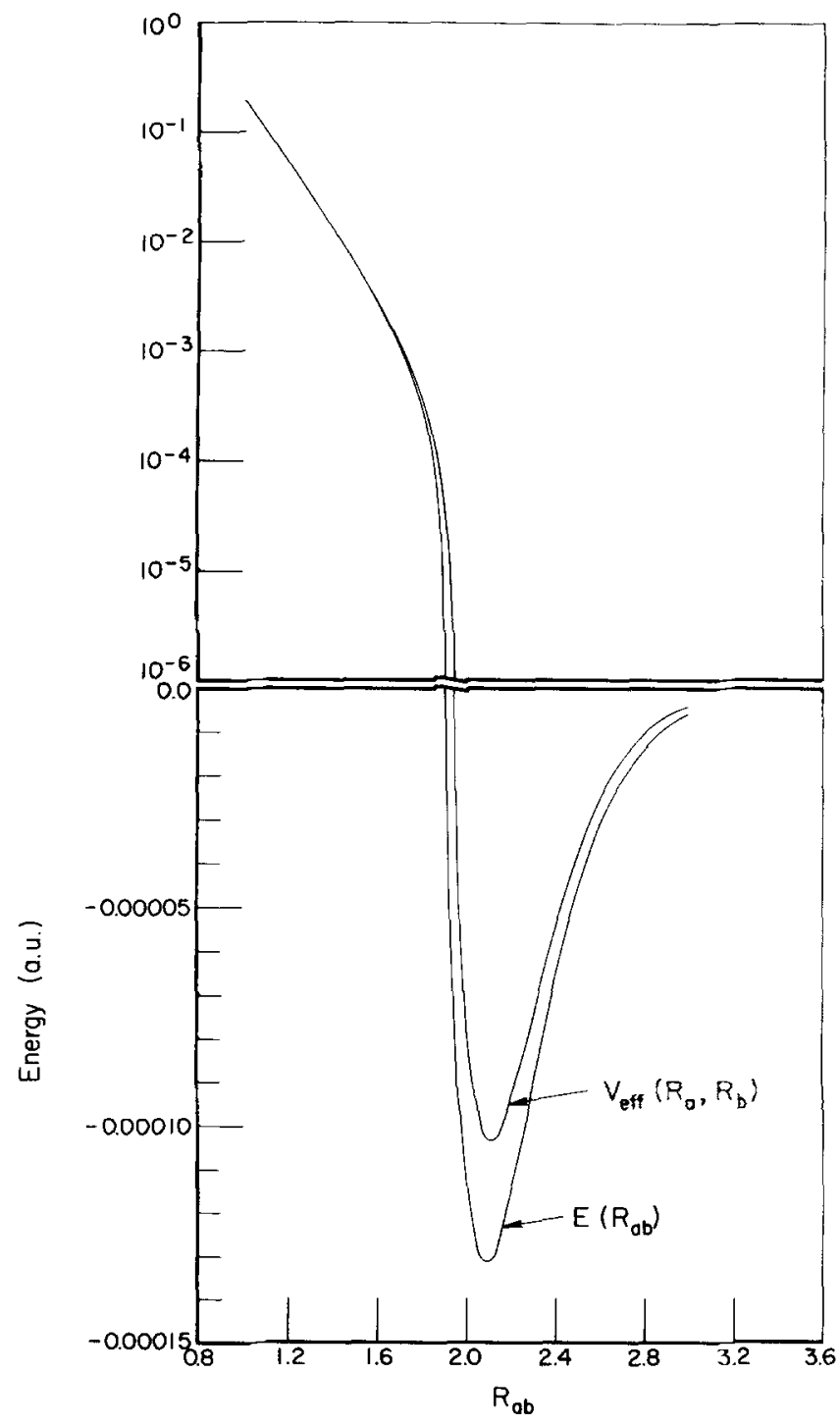

FIG. 9. The gas phase and effective neon-neon potentials are shown. The neon atoms are at 6.0 a.u. above the graphite surface oriented along the dashed line of Fig. 1. The distances are given in units of a graphite lattice carbon-carbon bond length.

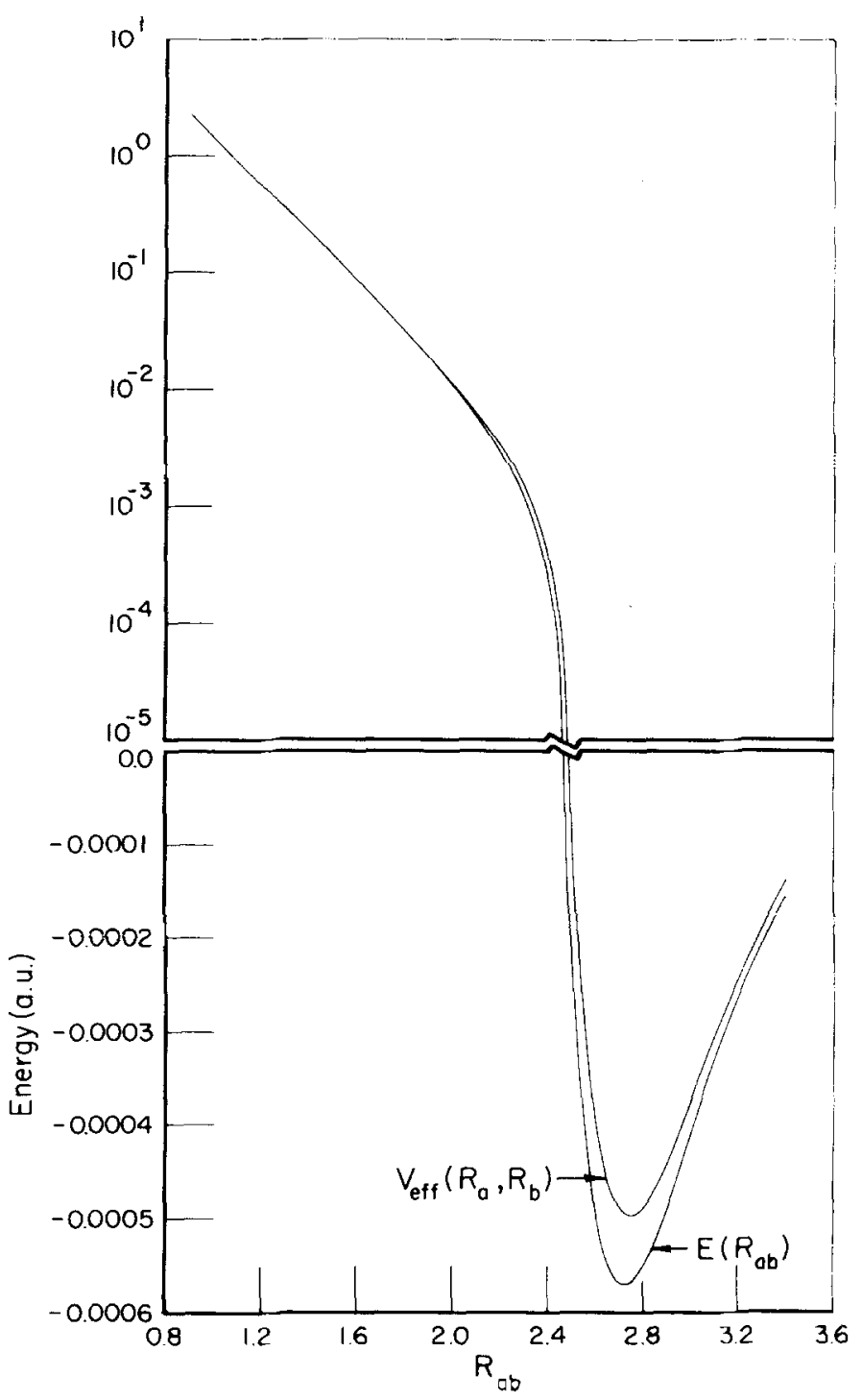

FIG. 10. The gas phase and effective krypton-krypton potentials are shown. The krypton atoms are at $6.8 \mathrm{a}, \mathrm{u}$. above the graphite surface oriented along the dashed line of Fig. 1. The distances are given in units of a graphite lattice carbon-carbon bond length.

separation, and $R_{a_{0}}$ and $R_{b_{0}}$ are the positions of the adatoms at equilibrium on the surface. In Table IV we compare the values of $\Delta$ computed in this calculation with the experimental values reported by Everett. ${ }^{8}$ The trend with respect to adatom size is correct, but the calculated values of $\Delta$ are too large. We expect that this discrepancy is mostly due to the approximate graphite density function used in this calculation. In I the graphite density function used was found to be deficient in the

TABLE IV. Theoretical and experimental values of $\Delta$.

\begin{tabular}{lll}
\hline \hline & $\Delta_{\text {eale }}$ & $\Delta_{\text {exp }}$ \\
\hline $\mathrm{Ne}$ & 0.214 & \\
$\mathrm{Ar}$ & 0.135 & 0.102 \\
$\mathrm{Kr}$ & 0.123 & 0.065 \\
\hline
\end{tabular}

"Reference 8. 
asymptotic tail. We expect that the errors introduced by the poor density function will have roughly the same effect on the contributions to the adatom-surface energy and the nonadditive energy. We therefore expect the importance of nonadditive effects relative to the total energy to be well described. To test this assumption, we computed the energy for $\mathrm{Ar}_{3}$ in a right isosceles triangular configuration. The minimum basis set described in I was used to generate the electron density for the argon atom at the $90^{\circ}$ vertex, and the extended basis of I was used for the other two argon atoms. The distance between the argon atom at the $90^{\circ}$ vertex and each of the other two argon atoms was taken to be 7 a.u. The same geometry was considered by $\mathrm{Kim} .{ }^{17} \mathrm{Kim}$ found a nonadditive contribution for this case of $0,0000104 \mathrm{a}$. $u$. and a total energy of $-0.000788 \mathrm{a}$.u. We found a nonadditive energy of $0.00000575 \mathrm{a}, \mathrm{u}$. and a total energy of -0.000347 a. u. In our case the nonadditive energy was $1.6 \%$ of the total, and in his case the nonadditive energy was $1.3 \%$ of the total. The relative importance of nonadditive effects are roughly the same in the two calculations, and we expect that the relative importance of nonadditive contributions to the total energy to be well described in our calculations. However, the relative importance of nonadditive effects with respect to the adatom-adatom interactions will probably not be accurate, because the unperturbed atom-atom GK potentials agree well with experiment. Thus, our computed values of $\Delta$ will be somewhat in error. We do not expect important errors in $\Delta$ owing to the improper treatment of the dispersion contributions. The dispersion error should affect all terms in the energy, and it should not have a large effect on the importance of nonadditive contributions relative to the gas phase potential.

\section{CONCLUSIONS}

It is clear from our results that rare gas films on graphite are highly mobile. The low migration barriers relative to adatom-adatom binding energies imply that these films are little perturbed by the details of the surface structure. Nonadditive effects modify the magnitudes of the adatom-adatom interactions, but not their relative orientations. It appears that potential energy considerations imply that the configuration of adsorbed atoms of neon, argon, and krypton will be out of register with the graphite substrate. In view of the conclusions of Ref. 2, this point requires further study.

Our GK results are valid only in the region of significant electronic overlap. This is an improvement over previous calculations which considered the nonadditive contributions to the dispersion energy. Our success in predicting the trends with respect to adatom size indi- cates the importance of these overlap effects.

The nonadditive contributions seem to have a moderating effect on the interaction potentials. In the strongly repulsive region the nonadditive effects decrease the repulsion, and the attraction is decreased in the attractive region. The nonadditive terms are an important fraction of the total energy only when the sum of the two-body energies becomes small. Since the total energy itself is very small in these regions, there are no real physical implications to the apparent dominance of the nonadditive terms, i.e., nonadditive effects make only a small change in the total energy potential surface. Of course, the nonadditive terms are important parts of the effective potential, and these effects have been seen experimentally.

\section{ACKNOWLEDGMENTS}

I would like to thank Dr. G. S. Painter and Professor D. E. Ellis for providing me with the graphite wavefunctions used in this work. I would like to thank Dr. C. W. Kern, Dr. I. Shavitt, and Dr. Y. S. Kim for many useful discussions, In particular, I would like to thank Dr. Shavitt for advice on the numerical aspects of this work and $\mathrm{Dr}$. Kim for providing the programs used to calculate the gas phase potentials.

*Present address: Department of Physics, The University of Utah, Salt Lake City, Utah 84112 .

${ }^{1}$ M. Bretz, J. G. Dash, D. C. Hickernell, E. O. Mclean, and O. E. Vilches, Phys. Rev. A 8, 1589 (1973).

${ }^{2}$ J. J. Lander and J. Morrison, Surf. Sci. 6, 1 (1967).

${ }^{3}$ M. P. Freeman, J. Phys. Chem. 62, 723 (1958).

${ }^{4}$ M. P. Freeman, J. Phys. Chem. 62, 729 (1958).

${ }^{5} J$. R. Sams, Jr., G. Constabaris, and G. D. Halsey, Jr., J. Chem. Phys. 36, 1334 (1962).

${ }^{6}$ J. A. Barker and D. H. Everett, Trans. Faraday Soc. 58, 1608 (1962).

${ }^{7}$ J. D. Johnson and M. L. Klein, Trans. Faraday Soc. 60, 1964 (1964).

${ }^{8}$ D. H. Everett, Discuss. Faraday Soc. 40, 177 (1965).

${ }^{9}$ R. Wolfe and J. R. Sams, J. Chem. Phys. 44, 2181 (1966).

${ }^{10} \mathrm{~J}$. D. Johnson and M. L. Klein, Trans. Faraday Soc. 63, 1269 (1967).

${ }^{11}$ J. D. Johnson, J. Phys. Chem. 72, 3697 (1968).

${ }^{12} \mathrm{O}$. Sir. noğlu and K. S. Pitzer, J. Chem. Phys. 32, 1279 (1960).

${ }^{13}$ A. D. McLachlan, Mol. Phys. 7, 381 (1964).

${ }^{14}$ T. B. MacRury and B. Linder, J. Chem. Phys. 54, 2056 (1971).

${ }^{15}$ D. L. Freeman, J. Chem. Phys, 62, 941 (1975).

${ }^{16}$ R. G. Gordon and Y. S. Kim, J. Chem. Phys. 56, 3122 (1972).

${ }^{17}$ Y. S. Kim, Phys. Rev. A 11, 796 (1975); 11, 804 (1975).

${ }^{18}$ A. J. Bennett, Phys. Rev. B 9, 741 (1974). 